\title{
INTERVIEW
}

For reprint orders, please contact: reprints@futuremedicine.com

\section{Searching for the footprints of a virus in lymphomas}

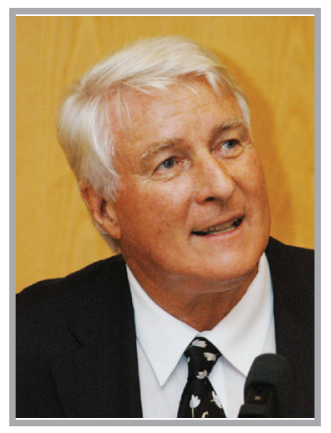

Volker Diehl* speaks with Sophie Breeze, Assistant Commissioning Editor: Professor Volker Diehl is Director Emeritus for Internal Medicine at the University of Cologne, Germany. Amongst numerous positions he was Founder and Chairman of the German Hodgkin Study Group (GHSG) for 30 years; he is now the Honorary Chairman of this group. He was Founding Director of the National Cancer Center in Heidelberg between 2004 and 2005. His major scientific achievements are: the first immortalization of umbilical cord blood cells to long-term lymphoblastoid cell lines; the first demonstration that Epstein-Barr virus causes infectious mononucleosis; the first establishment of Hodgkin-Reed Sternberg cell lines in vitro; the codiscovery of the $\mathrm{CD} 30$ antigen and codevelopment of associated monoclonal antibodies; and the initiator of the design of the BEACOPP principle in 1992. His clinical achievements are: the foundation of the GHSG, the design and establishment of six generations of consecutive Hodgkin trials within the GHSG; among other leading innovations the design and implementation of the BEACOPP principle for advanced Hodgkin lymphoma; the inauguration and establishment of the International Prognostic Index for advanced Hodgkin lymphoma together with Dirk Hasenclever; and the foundation of the European Task Force for Lymphocyte Predominant Hodgkin Lymphoma together with Harald Stein. His achievements have been recognized through the awarding of numerous prizes, most recently the American Society of Hematology (ASH) Award: Pioneer of Hematology (2009) and the ASH-Coulter-Award for Lifetime Achievement (2010).

\section{Q What sparked your interest}

in pursuing a career researching hematologic malignancies?

My scientific career started in 1966 as a virology research fellow in the Laboratory of Werner and Brigitte Henle at the Children's Hospital in Philadelphia (PA, USA), where I began to work with the Epstein-Barr virus (EBV) and was lucky enough, for the first time, to immortalize umbilical cord lymphocytes by adding EBV to continuously growing lymphoblastoid cell cultures. In 1967, I found, together with the Henles, that EBV causes infectious mononucleosis (IM). In 1968, on a grant from the American Cancer Society, I had the task of finding out whether IM also occurred around endemic Burkitt lymphoma cases in east Africa around Lake Victoria. Flying around with the Flying Doctor Service to these remote areas, together with Denis Burkitt, I found out that there is no IM in the Burkitt lymphoma endemic areas where malaria is holoendemic. Most of these children are already infested with the EBV at a very young age where their immune armamentarium is not able to build up a clinical syndrome like IM. As a first incentive for my interest in hematology was the work as a research fellow in the laboratory of the Henles in Philadelphia, USA, with a lymphotropic virus that was intimately related to blood cancers. Furthermore, later in Kenya, the close contact with clinicians at the Nairobi Kenyatta Hospital
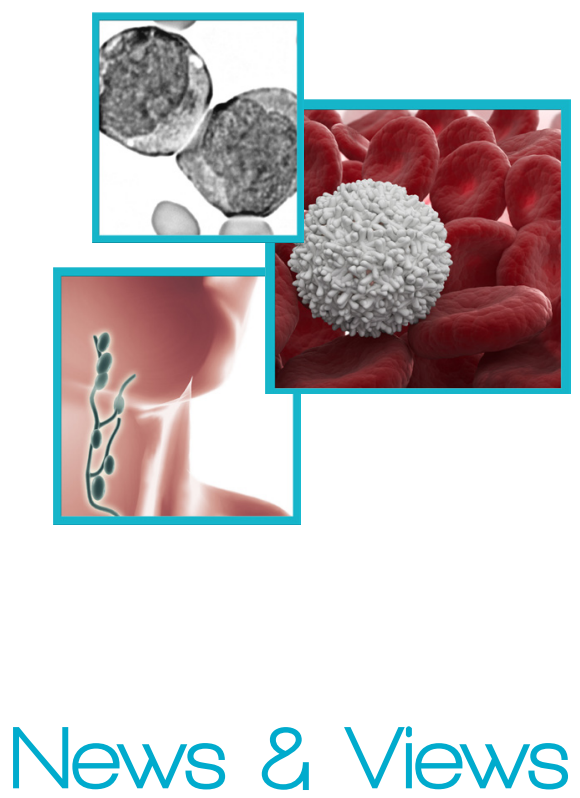

News

Journal Watch

Conference Scene

Interview

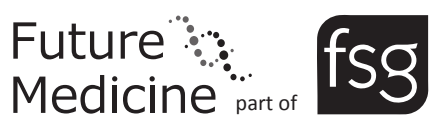


treating hematological malignancies made me interested and curious to get to know more about the etiology and pathogenesis of lymphomas and leukemias.

\section{Arguably one of your career} highlights was your codiscovery of the CD30 antigen, leading to the development of monoclonal antibodies. What impact has this discovery had on the way patients can expect to be treated now and in the future?

In 1978, I established the first Hodgkin Reed-Sternberg (HRS) cell line, L-428. In a collaboration with Stein, Lemke and Schwabe, we detected the CD30 antigen on these cells, assuming first that it was a Hodgkin-specific antigen but then finding out that EBV-transformed or phytoheme-agglutinin-stimulated cells also expressed this TNF family-related CD30 antigen that had never been described before. Stein and others produced monoclonal antibodies against this CD30 antigen. The naive antibodies did not show any significant cytotoxic effect against HRS cells. But then Peter Sentner, a biochemist from Seattle Genetics, succeeded in producing an antibody-drug conjugate (ADC) where the antibody was linked to a 'chemical bomb', auristatin, a tubulin inhibitor, to create a 'magic bullet' - a form of anticancer agent that Paul Ehrlich, the Nobel Prize winner in 1908 already visualized. This ADC has astonishing efficacy even in patients with multiple relapsed or refractory Hodgkin lymphomas (HLs) or anaplastic lymphoma lesions, resistant against all other strategies of radiation or chemotherapeutic agents. It is named brentuximab-vedotin, or Adcetris ${ }^{\circledR}$ (Seattle-Genetics, WA, USA), and is a real breakthrough in the fight against even highly resistant HLs, although it is not efficacious in every patient. Whether it will cure patients has yet to be determined in longer follow-up studies.

You have received numerous prizes in recognition of your achievements throughout your career, including the Pioneer of Hematology and the Award for Life Time Achievements from the American Society of Hematology. In an eventful career, what do you consider to be your greatest achievements?

It is hard to specify one career highlight, but for me one of the most important achievements in my scientific and clinical career was the in vitro transformation of human lymphocytes/umbilical cord blood cells for the first time ever by infection with EBV to immortalized lymphoblastoid cell cultures. Some other highlights for me include the original finding that EBV causes IM. The first in vitro cultivation of HRS cells L428, L540 and L1236, the design and clinical proof of the effectiveness of the bleomycin, etoposide, adriamycin, cyclophosphamide, oncovin, procarbazine and prednisone (BEACOPP) regimen for advanced $\mathrm{HL}$, and the establishment of a foundation for 'complementary medicine' and building the 'Haus LebensWert', as a center for 'complementary medicine' for patients with cancer at Cologne University (Germany). I am also very proud to have been the founder of the German Hodgkin Study Group (GHSG), the largest Hodgkin study group in the world.

\section{Q As the Founder and Chairman of} the GHSG, what do you think the key successes have been from the Hodgkin trials carried out by the group over the last 30 years?

The major success of the GHSG, which I founded in 1978, was to show that working together with all players on all levels of medical care for cancer patients and overcoming the selfishness of big 'egos' helps patients, doctors, nurses and medical students to foster progress in curing more patients and educating the medical staff and building a school of art for diagnostics, treatment and follow-up of patients with HLs. The next major accomplishment was the continuity of leadership and stepwise progress from one study generation to the next by creating new ideas on the results of the previous study generation in all strata of the HL risk groups. Over the past 30 years, the GHSG has been a valuable resource and driving force behind the establishment of a nationwide standard of the management of patients with HL, in Germany and in other European countries.
Since its establishment, the GHSG has been very effective in recruiting patients, and has recruited more than $60 \%$ of newly diagnosed HL patients into studies in Germany, treating more than $90 \%$ of HL patients in Germany according to the GHSG protocols. There have been over six generations of GHSG studies, which have reduced the amount of radiation in dosage, field size and number of patients needing radiation, as well as decreasing the intensity, drug quantity and cycle numbers of chemotherapy. With approximately 20,000 HL patients' data stored in the computer, the GHSG offers a precious resource for outcome, follow-up and toxicity evaluations on HL patient strategies.

\section{Q One of your research interests} includes the difference in therapy choices for early- and advanced-stage $\mathrm{HL}$. What would your recommendations be to physicians when treating $\mathrm{HL}$ ? The GHSG and the European Organisation for Research and Treatment of Cancer (EORTC) discriminate between three different risk groups of HL patients - early, intermediate and advanced - for treatment allocation, according to anatomical stage and biological and clinical risk factors. The risk factors that should be taken into account include extent of erythrocyte sedimentation rate, number of involved lymph node sites, large mediastinal mass and B symptoms (general symptoms in lymphomas, such as fever, nightsweat and weight loss). The earlyrisk group can be identified as being in stages I-II, A, B, without risk factors, while the intermediate risk group is also in the same stages but with risk factors. The advanced risk group can be identified as being in stages III-IV, A, B and IIB with large mediastinal mass. In terms of treatment strategies, the rationale for all stages is to reach the highest cure rate with the least possible short- and longterm toxicity. Therefore, the treatment strategies for each of these stages are quite different, and my recommendations are:

- Early stages: two adriamycin, bleomycin, velban, dacarbazin (ABVD) + 20 Gy involved-field irradiation (IFRT) 
- Intermediate stages: two escalated BEACOPP + two ABVD 30 Gy IFRT

- Advanced stages: six escalated BEACOPP \pm 30 Gy IFRT

\section{Speaking as a pioneer in $\mathrm{HL}$}

research, where do you think the future of innovative hematologic oncology research lies and how do you expect you will be involved with it?

In the future, we aim to be able to cure more than $95 \%$ of HL patients. Today, at least using the GHSG strategies, we achieve more than $90 \%$ tumor-free survival at 7 years with only one treatment application and not exposing the patients to the psychological and medical trouble of experiencing a first or second relapse. The experience of secondary neoplasias or cardiac damage due to the primary induction treatment (ABVD + HDCT + SCT vs escalated BEACOPP) is reduced for
BEACOPP and also needs less radiation. There are two major future aims that I believe should be the focus of HL research. The first is to be able to predict the risk of treatment failure at diagnosis with new diagnostic instruments, such as FDG-PET and other morphological and/or metabolic methods, or early discrimination, prior to treatment onset, of the molecular-genetic risk of failing therapy. The second is the development of personalized therapy, such as with ADCs like brentuximab-vedotin \pm chemo- or radio-therapy. Many new molecules and antibodies are coming to the market in the near future, and other anticancer modalities such as interfering with the intracellular pathways and signal transductions with miRNAs will possibly open new avenues for curing more HL patients, avoiding the hitherto overly toxic anticancer weapons like chemoand or radio-therapy. It is my hope that the GHSG will continue to work at the forefront of medical-scientific progress with gene technology laboratories and pharmaceutical companies, offering one of the best organized and clinically and bioinformatically experienced study groups in the world.

\section{Disclaimer}

The opinions expressed in this interview are those of the interviewee and do not necessarily reflect the views of Future Medicine Ltd.

\section{Financial \& competing interests}

disclosure

$V$ Diehl is a consultant for Seattle-Genetics but no stocks or other interests in this company or other companies. $V$ Diehl has no other relevant affliations or financial involvement with any organization or entity with a financial interest in or financial conflict with the subject matter or materials discussed in the manuscript apart from those disclosed.

No writing assistance was utilized in the production of this manuscript. 